Nonlinear Processes in Geophysics, 12, 849-861, 2005

SRef-ID: $1607-7946 / \mathrm{npg} / 2005-12-849$

European Geosciences Union

(c) 2005 Author(s). This work is licensed

under a Creative Commons License.

\title{
On the predictability of ice avalanches
}

\author{
A. Pralong ${ }^{1}$, C. Birrer ${ }^{2}$, W. A. Stahel ${ }^{2}$, and M. Funk ${ }^{1}$ \\ ${ }^{1}$ Laboratory of Hydraulics, Hydrology and Glaciology, Swiss Federal Institute of Technology, 8092 Zürich, Switzerland \\ ${ }^{2}$ Seminar for Statistics, Swiss Federal Institute of Technology, 8092 Zürich, Switzerland
}

Received: 13 June 2005 - Revised: 19 August 2005 - Accepted: 19 August 2005 - Published: 30 September 2005

\begin{abstract}
The velocity of unstable large ice masses from hanging glaciers increases as a power-law function of time prior to failure. This characteristic acceleration presents a finite-time singularity at the theoretical time of failure and can be used to forecast the time of glacier collapse. However, the non-linearity of the power-law function makes the prediction difficult. The effects of the non-linearity on the predictability of a failure are analyzed using a non-linear regression method. Predictability strongly depends on the time window when the measurements are performed.

Log-periodic oscillations have been observed to be superimposed on the motion of large unstable ice masses. The value of their amplitude, frequency and phase are observed to be spatially homogeneous over the whole unstable ice mass. Inclusion of a respective term in the function describing the acceleration of unstable ice masses greatly increases the accuracy of the prediction.
\end{abstract}

\section{Introduction}

The prediction of ice avalanches from hanging glaciers is based on the progressive acceleration observed on large unstable ice masses prior to their collapse. A suitable model of the observed acceleration presents a finite time singularity; that is, the velocity tends to infinity as the time approaches a finite time. This finite time corresponds to the time of failure.

Finite time singularity models have been used for characterizing a large variety of phenomena. Rheologists have suggested such models to describe the ductile fracture of samples of rock, soil, high-performance metal alloys, concrete, polymers and ice (see Varnes, 1983 for a review and Voitkovskii, 1960; Szyszkowski and Glockner 1986; Mahrenholtz and Wu 1992 for laboratory ice). At large scales, finite time singularity models have been proposed to describe the mechanisms of landslides (e.g. Crosta and

Correspondence to: M. Funk

(funk@vaw.baug.ethz.ch)
Agliardi, 2003; Amitrano et al., 2005), earthquakes ${ }^{1}$ (e.g. Bufe and Varnes, 1993; Bowman et al., 1998), volcanic eruptions (e.g. Voight, 1988), fracture of structures (e.g. Johansen and Sornette, 2000), inflation (Sornette et al., 2003), finance, economy, population (Johansen and Sornette, 2001) and break-off of ice chunks from hanging glaciers (Haeberli, 1975; Flotron, 1977; Iken, 1977; Röthlisberger, 1981; Lüthi, 2003; Pralong and Funk, 2005a,b).

Finite-time singularities are caused by positive feedback processes, which lead to a catastrophic evolution of the observed quantities. Sammis and Sornette (2002) reviewed positive feedbacks involved in the rupture of materials; Sornette et al. (2003) mentioned a positive feedback involved in inflation.

A suitable model for such catastrophic evolutions is given by Voight's differential equation (Voight, 1988)

$\ddot{f}=A(\dot{f})^{\alpha}$,

where the dot denotes the time derivative and $f(t ; A, \alpha$, $\left.c_{1}, c_{2}\right)$ is the function describing the temporal evolution of a measured quantity. Observations $Y_{i}$ are obtained at times $t_{i}$. They include a random disturbance $Z_{i}$, i.e.

$Y_{i}=f\left(t_{i} ; A, \alpha, c_{1}, c_{2}\right)+Z_{i}$.

$A$ and $\alpha>1$ are the parameters describing the catastrophic evolution of $Y$ and $c_{1}, c_{2}$ are the two integration constants.

\footnotetext{
${ }^{1}$ This topic is controversial. Helmstetter et al. (2003) argued that the progressive acceleration of representative seismic quantities is observed only by stacking many sequences of seismic activity and results from a different mechanism than critical phenomena. Zöller and Hainzl (2002) claimed that the acceleration of seismicity observed before large earthquakes may be spurious. They showed that there is a $20 \%$ probability of observing the same acceleration by chance in a synthetic catalog of random earthquakes. For these reasons the presence of finite-time singularities in earthquakes (i.e. the use of accelerating precursory seismicity to predict large earthquakes) is questionable.
} 

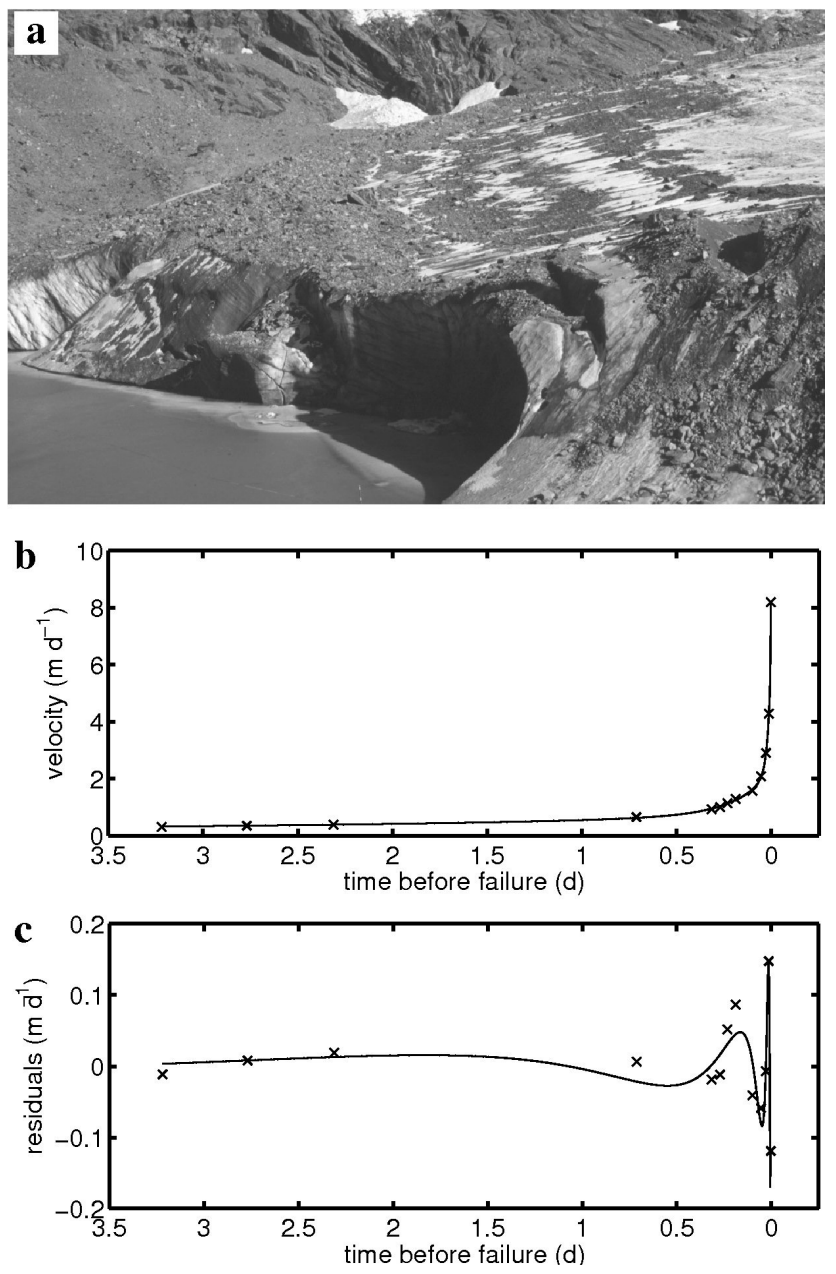

Fig. 1. Data set of Gruben glacier, Switzerland. (a) Photo of a calving event similar to the one measured by Haeberli (1975). The unstable mass is visible in the foreground. (b) Measured relative velocity $\dot{Y}$ (Haeberli, 1975) versus time (crosses) and its associated fit (solid line) based on Eq. (6). The estimated parameters $\hat{\theta}_{i}$ of Eq. (6) are listed in Table 1. The predicted failure time (corresponding to abscissa zero) was 9 September 1974. (c) Residuals of the fit. The solid line indicates the fit of the log-periodic oscillations (see Sect. 5).

Integrating Eq. (1) for $\alpha>1$ and assuming that $\dot{f}$ at the time of the singularity is infinite, leads to (Voight, 1988)

$f(t, \boldsymbol{\theta})=\left\{\begin{array}{lll}\theta_{4}-\theta_{3} \ln \left(\theta_{1}-t\right) & \text { if } & \theta_{2}=0 \\ \theta_{4}-\frac{\theta_{3}}{\theta_{2}}\left(\theta_{1}-t\right)^{\theta_{2}} & \text { if } & \theta_{2} \neq 0\end{array}\right.$,

with $\theta_{1}$ the time of failure, $\theta_{4}$ a constant and

$\theta_{2}=\frac{\alpha-2}{\alpha-1}<1 \quad$ and $\quad \theta_{3}=(A(\alpha-1))^{\theta_{2}-1}$.

For the failure of hanging glaciers, observations and numerical simulations (Haeberli, 1975; Iken, 1977; Pralong and Funk, 2005a) show that the relative motion of an unstable ice mass (relative to the motion of the stable glacier part located directly upstream of the unstable part) is adequately described by Eq. (1); that is, the relative motion of the unstable ice masses can be modeled by $f^{2}$.

Two different approaches can be applied in order to predict the time of failure $\theta_{1}$ : a "rheological" and an "empirical" approach. The rheological approach considers Eq. (1) (or a similar equation) as a constitutive relation for the evolution of $Y$ and looks for general relations for the parameters $A=A(\sigma, T, \ldots)$ and $\alpha=\alpha(\sigma, T, \ldots)$, which may depend, for example, on the stress $\sigma$ and the temperature $T$. An a priori knowledge of $A$ and $\alpha$ (or equivalently of $\theta_{2}$ and $\theta_{3}$ ) permits then to estimate the time of failure $\theta_{1}$. For example, by setting $A=B(T) \sigma^{r}, \alpha=k+2$ and $\dot{Y}=1 /(1-D)$, where $B(T)$ is a function of temperature, $r$ and $k$ are material parameters and $D$ is the classical damage variable of the continuum damage mechanics (e.g. Lemaitre, 1996), Voight's equation (Eq. 1) reduces to

$\dot{D}=B(T) \sigma^{r}(1-D)^{-k}$.

This equation is the classical Kachanov-Rabotnov constitutive relation (Kachanov, 1957; Rabotnov, 1969), modeling the accumulation of isotropic damage in material subject to uniaxial load. Equation (5) describes, therefore, a finite time singularity if $k>-1$ (i.e. $\alpha>1$ ). The rheological approach is appropriate for describing the fracture of homogeneous samples of ductile materials; however, a precise prediction cannot be obtained. The application of this method to the description of the failure of large-scale structures by the integration of a local damage evolution law (e.g. Eq. 5) in a large-scale domain can lead to an adequate capture of the physics of the global fracture (e.g. Lyakhovsky et al. (2001) for earthquakes, and Pralong and Funk (2005a) for fracture processes in glaciers), but fails to predict accurately the time of the global failure, since the conditions prevailing before the failure process are largely unknown and the parameters are subject to uncertainties.

In the empirical approach, in contrast to the rheological approach, $A$ and $\alpha$ (or $\theta_{2}$ and $\theta_{3}$, respectively) are not a priori determined. The prediction of the failure time $\theta_{1}$ is thus a fitting problem of measured data, where the critical quantity $Y$ is compared to the solution of Voight's equation, and the parameters of Eq. (3) and especially $\theta_{1}$ are estimated. This approach turns out to be more precise than the rheological approach, since the a priori informations needed for the rheological approach are affected by uncertainties. This approach is usually applied to the prediction of the singularity of large-scale processes, which can cause great damage. In such a case, precise prediction allows for preventive actions. This paper focuses on the empirical approach applied to the destabilization of ice chunks from hanging glaciers.

\footnotetext{
${ }^{2}$ In some particular failure processes, which are not considered in this paper, the absolute motion of the unstable ice masses is modeled by $f$ (Pralong and Funk, 2005b).
} 


\section{Measurements}

The motion of several unstable ice masses was monitored by the Laboratory of Hydraulics, Hydrology and Glaciology (VAW) of the Swiss Federal Institute of Technology Zürich (ETHZ) within the scope of hazard assessment or research programmes. Of the various data sets collected, three will be considered here. The others do not contain enough measurements or are affected by a scattering that is too broad to be useful.

The first data set describes the relative motion of a calving ice mass (Figs. 1a and b) measured by Haeberli (1975) at Gruben glacier, Switzerland. The measurement equipment was a wire fixed at one end to the unstable ice mass and at the other end to a dial gauge attached to the stable part of the glacier. The time of failure was registered. Haeberli considered the relative velocity $\dot{Y}$ (time derivative of the motion $Y$ ) of the unstable ice mass instead of the relative motion $Y$. The function used to describe $\dot{Y}$ is thus the time derivative of Eq. (3):

$\dot{f}(t, \boldsymbol{\theta})=\theta_{3}\left(\theta_{1}-t\right)^{\theta_{2}-1}$.

The second data set corresponds to the acceleration of an unstable ice mass measured by the authors in 2001 at the front of the Eiger hanging glacier, Switzerland (Figs. 2a and $b$ ). The measurement equipment was a theodolite laserdistometer installed at Eiger glacier (a fixed position near the glacier) and one reflector mounted on a stake drilled into the unstable ice mass. Reference reflectors installed on a rock face close to the unstable ice mass enabled the correction of the measurements, which are influenced by meteorological conditions. For this data set, only the absolute motion (denoted by $Y^{\mathrm{a}}$ ) is known. The motion of the stable glacier part upstream of the unstable ice mass was not measured. It is assumed that during the measurements, the velocity of the stable glacier part is constant. The function which models the motion $Y^{\mathrm{a}}$ reads

$f^{\mathrm{a}}(t, \boldsymbol{\theta})=\left\{\begin{array}{lll}\theta_{5} t+\theta_{4}-\theta_{3} \ln \left(\theta_{1}-t\right) & \text { if } & \theta_{2}=0 \\ \theta_{5} t+\theta_{4}-\frac{\theta_{3}}{\theta_{2}}\left(\theta_{1}-t\right)^{\theta_{2}} & \text { if } & \theta_{2} \neq 0\end{array}\right.$,

with $\theta_{5}$ the constant velocity of the upstream glacier part. The time of failure of the unstable mass is not known as a subfailure occurred prior to the main failure, and caused the measurement equipment on the glacier to be lost.

The third data set describes the motion of several material points (stakes with reflectors) installed on a single unstable ice mass at the front of the Mönch hanging glacier, Switzerland (Fig. 3a). The measurements were performed by the authors in 2003, with the same equipment as for the Eiger hanging glacier. The three material points used for the analysis correspond to points 1, 2 and 3 of Fig. 3a. Points 4 and 5 present a temporal shift of the beginning of the acceleration (relatively to points 1, 2 and 3) and points 6 and 7 showed no acceleration during the period of measurement. The motion of point 1 is shown in Fig. $3 \mathrm{~b}$ as an example. The time of failure of the unstable ice mass is unknown, for the same rea-
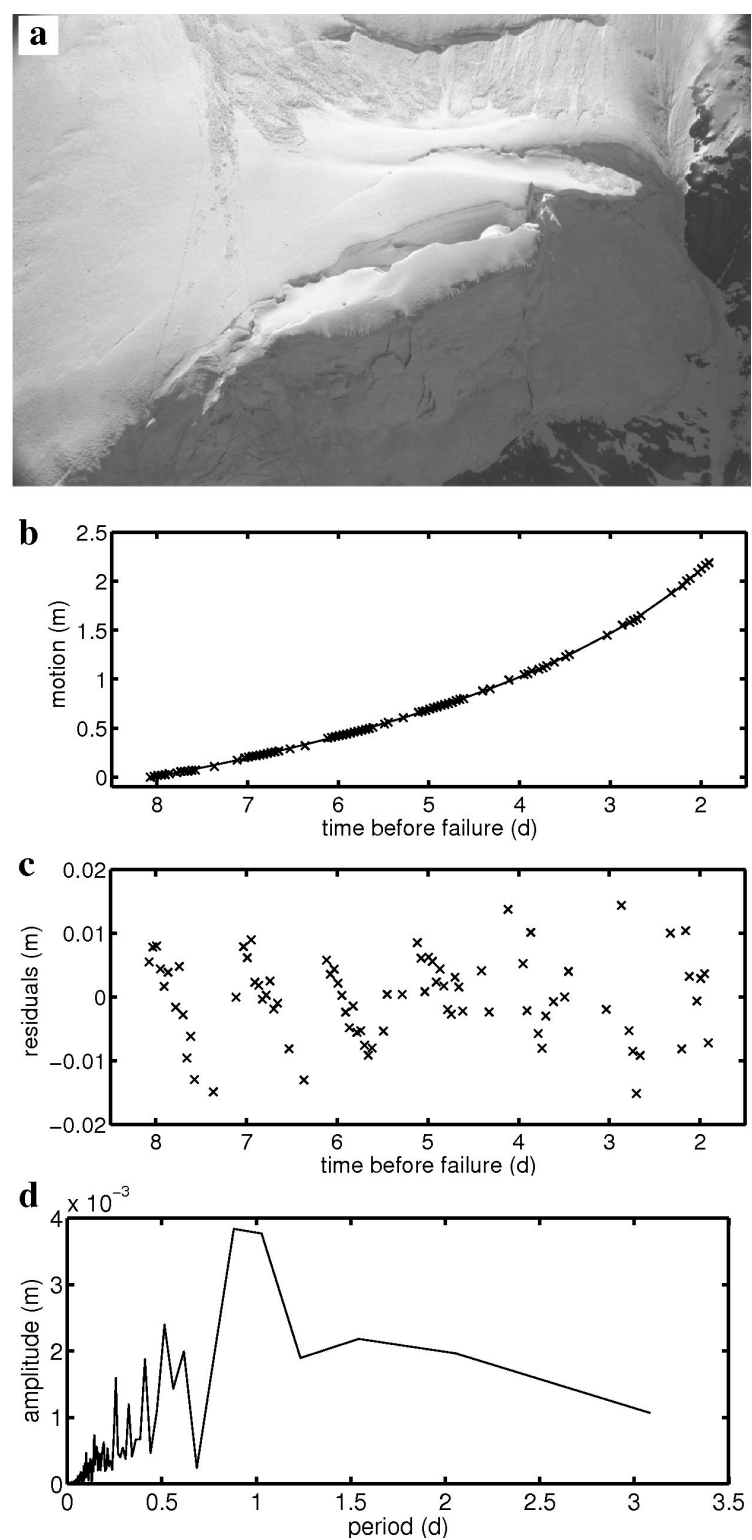

Fig. 2. Data set of Eiger glacier, Switzerland. (a) Photo of the measured unstable ice mass. The unstable mass is approximately $60 \mathrm{~m}$ high, $150 \mathrm{~m}$ long (direction normal to the ice flow) and $30 \mathrm{~m}$ wide. (b) Motion $Y^{\mathrm{a}}-\theta_{5} t$ versus time (crosses) and its associated fit (solid line) based on Eq. (7). The estimated parameters $\hat{\theta}_{i}$ of Eq. 7 are listed in Table 1. The predicted failure time (corresponding to abscissa zero) was 20 August 2001. (c) Residuals of the fit. d) Fourier analysis of the residuals.

son as for the Eiger measurements ${ }^{3}$. The measurements were affected by slight variations in the position of the theodolite

\footnotetext{
${ }^{3}$ Here only equipment at points 1 and 2 were lost. But the secondary failure, which led to this loss, reduced the mass of the unstable ice chunk, relaxed the stresses responsible for the destabilization process and thus induced a discontinuity in the acceleration of point 3. After that event, the unstable ice mass continued to fall in successive partial beaks (the measurements of the other points gave therefore no better results).
} 


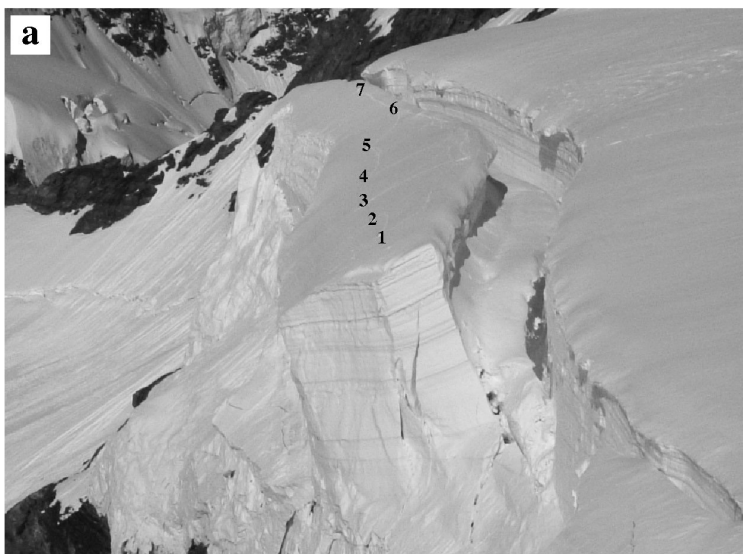

b

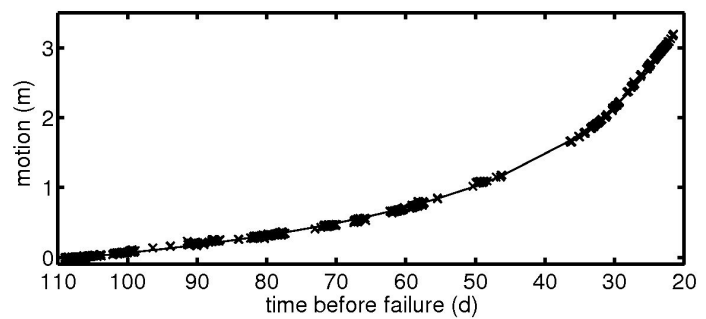

c

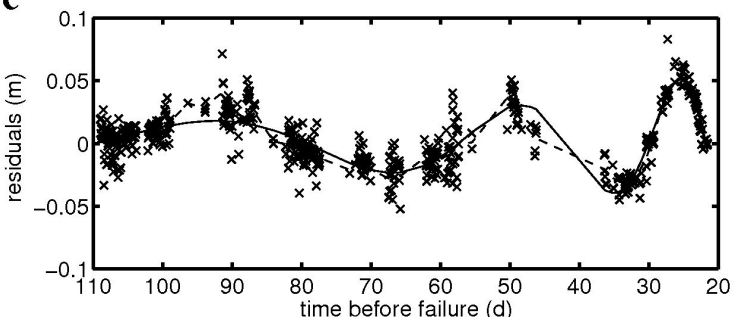

d

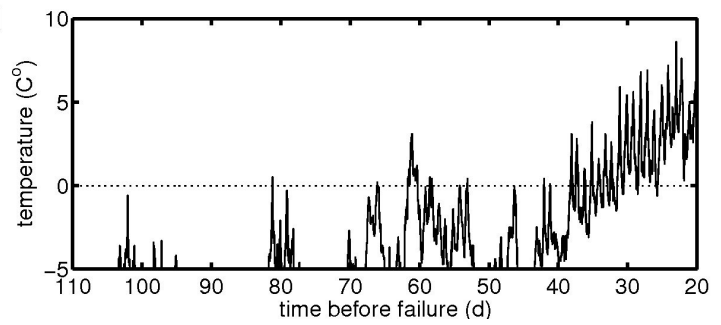

Fig. 3. Data set of Mönch glacier, Switzerland. (a) Photo of the measured unstable ice mass. The numbers indicate the location of the measured points. The unstable mass is approximately $50 \mathrm{~m}$ high, $300 \mathrm{~m}$ long (direction normal to the ice flow) and $40 \mathrm{~m}$ wide. The distance between points 1, 2 and 3 amounts to approximately $30 \mathrm{~m}$. (b) Motion $Y^{\mathrm{a}}-\theta_{5} t$ versus time (crosses) of point 1 and its associated fit (solid line) based on Eq. (7). The estimated parameters $\hat{\theta}_{i}$ of Eq. (7) are listed in Table 1. The predicted failure time (corresponding to abscissa zero) was July 4, 2003. (c) Residuals of the fit. The solid line indicates the fit of the log-periodic oscillations (see Sect. 5). The dashed line shows the smooth cruve of the residuals. (d) Air temperature at Jungfraujoch (MeteoSwiss data) located one kilometer from the glacier (solid line). The dotted line depicts the ice melting point.

(Appendix A). To account for these variations, the position of the theodolite was calculated at each measurement cycle from the position of four reference reflectors located on rock faces around the theodolite (Appendix A). Again, only the absolute motion $Y^{\mathrm{a}}$ is known. It is modeled by Eq. (7). It will again be assumed that the velocity of the stable glacier part is constant. For this data set, this assumption is questionable, since a significant increase in the air temperature above the melting point occurred during the failure process (Fig. 3d), and could have caused an acceleration of the glacier, thereby modifying the velocity $\theta_{5}$. The measurements revealed a variation in the velocity $\dot{Y}^{\text {a }}$ of points 4 and 5 which could be related either to air temperature ${ }^{4}$ or to the beginning of the destabilization process. For points 1, 2 and 3, the measurements did not reveal variations in the velocity which could be related to air temperature.

The estimates of the parameters $\theta_{i}$ (using a least squares method) for the failure of the three different glaciers are reported in Table 1. The residuals from the fits are shown in Figs. 1c, 2c and 3c. For the Gruben data set, the estimated failure time $\theta_{1}$ occurred some minutes before the observed failure. The residuals of the Eiger data set show strong oscillations. The Fourier analysis (Fig. 2d) revealed a dominant frequency corresponding to one day. Since the absolute motion $Y^{\mathrm{a}}$ is considered, these oscillations can be associated to the daily fluctuations of the basal sliding. Such fluctuations are commonly observed on glaciers (e.g. Sugiyama and Gudmundsson, 2003). The residuals of the Mönch data set show clear log-periodic oscillations. This behavior will be discussed in Sect. 5. Because of the few data points, the residuals of the Gruben data set do not allow to validate the presence of log-periodic oscillations. Nevertheless, the data set has been tentatively fitted by using the model with logperiodic oscillations ${ }^{5}$.

\section{Non-linear regression analysis}

The aim of this section is to present a method to obtain estimates of the parameters of the non-linear function 3 and their confidence intervals. The data set of the Gruben glacier is considered for illustration (Fig. 1).

The fitting process should account for the fact that the failure time must be greater than the time of the last observation;

\footnotetext{
${ }^{4}$ The following model is considered to support the analysis of the dependence of the glacier velocity to the air temperature. A linear water reservoir model (e.g. Hock and Noetzli, 1997) is used to estimate the water level in the glacier. The water supply of the reservoir is the water resulting from the melt of the snow covering the glacier. The melting rate is estimated with the air temperature. The water level can then be related to the basal sliding and the glacier velocity (e.g. Sugiyama and Gudmundsson, 2003).

${ }^{5}$ The increase of the noise amplitude at the end of the time series (Fig. 1c) does not only result from the smaller sampling time, which magnifies the noise of the derivative. Indeed, the inaccuracy of the measurements, which amounts according to Haeberli (1975) to approximatively $0.3 \mathrm{~mm}$, leads to a value of the root-mean-square error of approximatively $0.025 \mathrm{md}^{-1}$. This is two times smaller than the error of the fit without oscillations and similar to the error of the fit with oscillations (see Table 1).
} 
Table 1. Values of the estimated parameters $\hat{\theta}_{i}$ for the five data sets analyzed in this paper. The values of $\hat{\theta}_{2}$ to $\hat{\theta}_{5}$ in the upper part of the table are identified by using the model without log-periodic oscillations (Eq. 6 or 7). The values of $\hat{\theta}_{1}$ to $\hat{\theta}_{8}$ in the lower part of the table are identified by using the model with log-periodic oscillations (Eq. 12 or 13, see Sect. 5). $\theta_{1}$ and $\theta_{4}$ are integration constants. They depend on the value of $t_{0}$ and $Y\left(t_{0}\right)$, and do not influence the shape of the acceleration (the differential Eq. (1) depends only on $\alpha$ and $A$; that is not on $\theta_{1}$ and $\theta_{4}$ ). They are thus not reported in this table. Only the values of $\hat{\theta}_{1}$ corresponding to the measurements at Gruben and Mönch are reported for discussion (see text). The value of the estimated parameter of the steady motion $\left(\hat{\theta}_{5}\right)$ vanishes for the measurement at Gruben, since the relative motion of the unstable ice mass was measured. The values of the parameters corresponding to the model with log-periodic oscillations have not been determined for the measurements at Eiger, since no oscillations were observed (see text). The root-mean-square error (rmse) is also reported for the fits with and without log-periodic oscillations. The unit $\mathrm{md}^{-1}$ refers to the Gruben data set (velocity of the unstable ice mass) and the unit $\mathrm{m}$ to the other data sets (displacement of unstable ice masses).

\begin{tabular}{lllllll}
\hline Parameter & Units & Gruben & Eiger & Mönch $_{1}$ & Mönch $_{2}$ & Mönch $_{3}$ \\
\hline$\hat{\theta}_{1}$ & $\mathrm{~d}$ & 12.490 & & 120.81 & 135.59 & 141.77 \\
$\hat{\theta}_{2}$ & - & 0.523 & -0.110 & -1.573 & -2.567 & -2.858 \\
$\hat{\theta}_{3}$ & $\mathrm{md}^{-\theta_{2}}$ & 0.545 & 1.770 & $1.28 \mathrm{e} 3$ & $1.38 \mathrm{e} 5$ & $5.61 \mathrm{e} 5$ \\
$\hat{\theta}_{5}$ & $\mathrm{md}^{-1}$ & 0 & 0.039 & 0.102 & 0.106 & 0.105 \\
rmse & $\mathrm{md}^{-1} / \mathrm{m}$ & 0.055 & 0.007 & 0.020 & 0.022 & 0.021 \\
\hline$\hat{\theta}_{1}$ & $\mathrm{~d}$ & 12.493 & & 108.54 & 106.53 & 108.00 \\
$\hat{\theta}_{2}$ & - & 0.534 & & -0.749 & -0.685 & -0.879 \\
$\hat{\theta}_{3}$ & $\mathrm{md}^{-\theta_{2}}$ & 0.554 & & 32.50 & 21.31 & 37.90 \\
$\hat{\theta}_{5}$ & $\mathrm{md}^{-1}$ & 0 & & 0.098 & 0.101 & 0.102 \\
$\hat{\theta}_{6}$ & - & 0.030 & & 0.013 & 0.014 & 0.020 \\
$\hat{\theta}_{7}$ & $\log (\mathrm{d})$ & 0.090 & & 0.505 & 0.510 & 0.537 \\
$\hat{\theta}_{8}$ & - & 1.095 & & 0.469 & 5.116 & 2.722 \\
rmse & $\mathrm{md}^{-1} / \mathrm{m}$ & 0.032 & & 0.012 & 0.013 & 0.013 \\
\hline
\end{tabular}

that is, $\theta_{1}>\max \left(t_{i}\right)$. Moreover, according to Eq. $4, \theta_{2}<1$. The following parameter transformations force the parameters $\theta_{i}$ to satisfy these constraints:

$\theta_{1}=\max \left(t_{i}\right)+\exp \left(\phi_{1}\right), \quad \theta_{2}=1-\exp \left(\phi_{2}\right), \quad \theta_{3}=\phi_{3},(8)$

where $\boldsymbol{\phi}$ is the new parameter set of $\dot{f}$.

The subplots on the diagonal of the Fig. 4 are the profile- $t$ plots, the other subplots are the profile traces. The profile- $t$ plots show the dependence of the profile- $t$ function $\tau_{k}$ on the parameters $\phi_{k}$ (solid line). $\tau_{k}$ is the signed square root of the likelihood ratio test statistic for a null hypotheses about $\phi_{k}$ (Appendix B; Bates and Watts, 1988). In comparison, the test statistic based on the linear approximation of the regression in $\hat{\boldsymbol{\phi}}$ (the estimate $\hat{\boldsymbol{\phi}}$ is depicted by a cross) is displayed using a dash-dotted line. It is linear in $\phi_{k}$. The confidence interval derived from $\tau$ (Appendix B) is depicted by dashed lines, and the confidence interval derived from the linearized statistic test by dotted lines. Since the linear approximations are excellent, the difference can barely be seen.

The off-diagonal diagrams display the profile traces (Appendix B; Bates and Watts, 1988). They represent the correlations between the different parameters $\phi_{i}$ in the vicinity of $\hat{\boldsymbol{\phi}}$. In each profile subplot, the closer the two lines are to each other, the more the parameters of the subplot are correlated.

\section{Sensitivity analysis}

This section attempts to determine the influence of the measurement scheme on the accuracy of the prediction. The accuracy of the prediction is quantified by the size of the confidence interval for the time of failure.

\subsection{Method}

The set of the measurement times $\left(t_{1}, \ldots, t_{n}\right)$ and the accuracy of the measurements are fixed by the parameter set $\mathcal{S}$

$\mathcal{S}=\left(\sigma_{Y}, \delta_{Y}, \Delta t_{\mathrm{E}}, \Delta t_{\mathrm{B}}\right)$,

where $\sigma_{Y}$ is the standard deviation of the measurements, $\delta_{Y}$ is the periodicity of the measurements, $\Delta t_{\mathrm{E}}=\theta_{1}-t_{\mathrm{E}}$ is the time span between the end of the measurements (at time $t_{\mathrm{E}}$ ) and the failure (at time $\theta_{1}$ ) and $\Delta t_{\mathrm{B}}=\theta_{1}-t_{\mathrm{B}}$ is the time span between the beginning of the measurements (at time $t_{\mathrm{B}}$ ) and the failure.

In order to analyze the sensitivity of the accuracy of the prediction to the parameters $\mathcal{S}_{i}$, a collection of synthetic data sets is created with Eq. (3) by using the values of the parameters $\theta_{i}$ identified for three analyzed break-off events (Gruben, Eiger, Mönch ${ }_{1}$; Table 1) and with different sets of measurement times and different accuracies of measurements (corresponding to different values of $\mathcal{S}_{i}$ ). A synthetic Gaussian noise is assumed according to the value of the parameter $\sigma_{Y}$. 

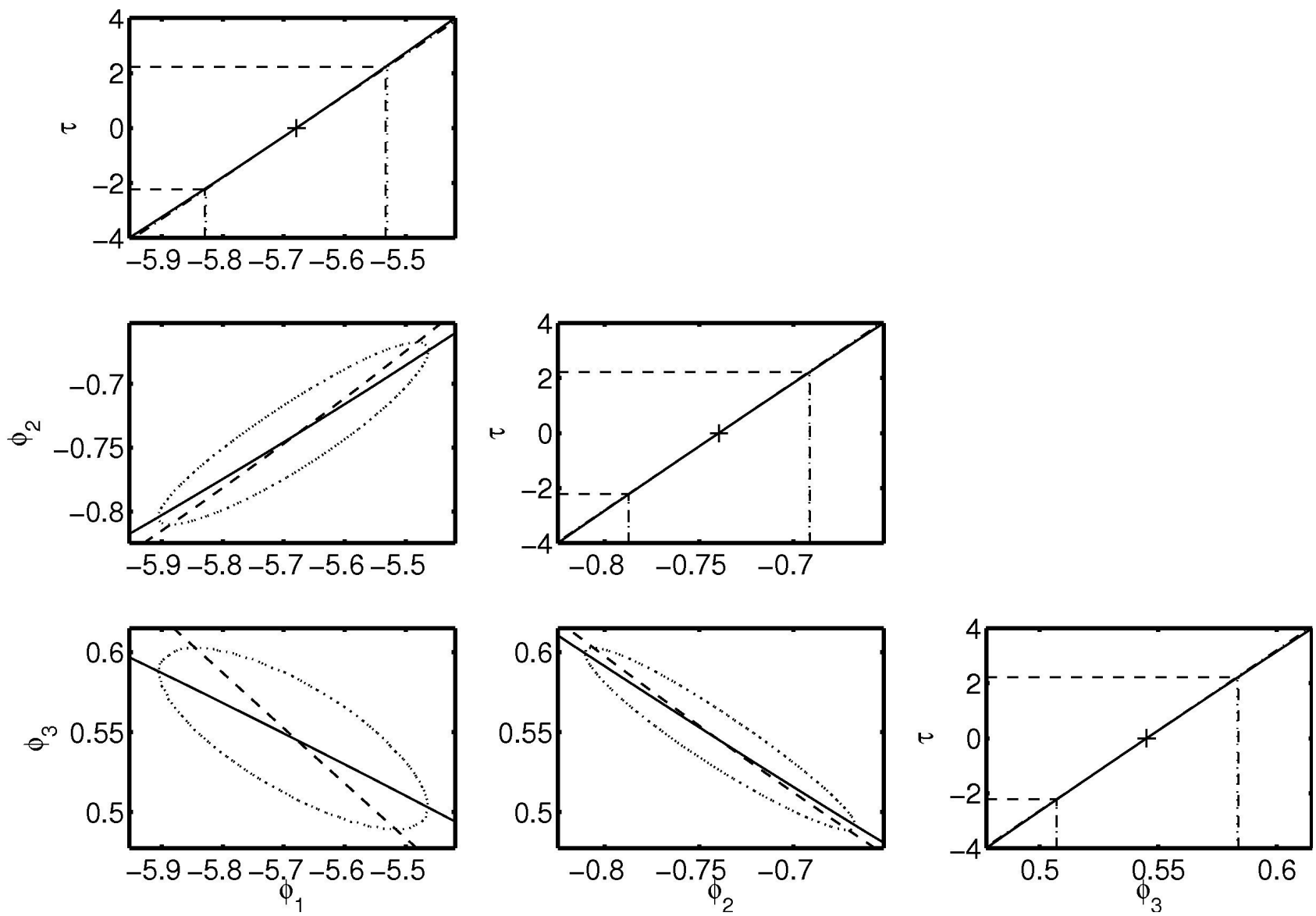

Fig. 4. Non-linear regression analysis of the Gruben data set (see text). The diagonal diagrams show the profile- $t$ plots. The $95 \%$ confidence interval of each parameter is indicated by dashed lines. The off-diagonal diagrams show the profile traces. The $95 \%$ confidence contours (dotted lines) of the pair-parameters are also shown.

The periodicity of the measurements is supposed to be constant with time and to equal $\delta_{Y}$. In this section, we assume no further disturbances (like daily variations of $\theta_{5}$ or $\log$ periodic oscillations), even though they could influence the accuracy of the forecast. The disturbance $Z$ has, therefore, a constant centered normal distribution and no time correlation.

The sensitivity analysis consists in letting the parameters $\mathcal{S}_{i}$ vary independently around defined reference values $\mathcal{S}_{i}^{*}$ and analyzing the effect of these variations on the confidence interval of $\theta_{1}$ by using the non-linear regression analysis presented in Sect. 3. The reference values $\mathcal{S}_{i}^{*}$ are set at

$\mathcal{S}^{*}=\left(\sigma_{Y}^{*}=0.01 \mathrm{~m}, \delta_{Y}^{*}=0.05 \mathrm{~d}, \Delta t_{\mathrm{E}}^{*}=3 \mathrm{~d}, \Delta t_{\mathrm{B}}^{*}=30 \mathrm{~d}\right)$.

The value $\sigma_{Y}^{*}$ is given by the accuracy of today's theodolite laser-distometers ${ }^{6} . \delta_{Y}^{*}$ corresponds approximately to one measurement per hour. Experience has shown that a precise prediction emerges only a few days before the effective collapse. Therefore, $\Delta t_{\mathrm{E}}^{*}$ is chosen as three days. $\Delta t_{\mathrm{B}}^{*}$ corresponds to a time series of measurements (until failure) of one

\footnotetext{
${ }^{6}$ This accuracy is obtained by normalizing each measurement with reference measurements (in a similar way to the method used for differential GPS measurements).
}

month. The unstable ice chunks are regularly losing mass by fracture during the acceleration process. If the detached mass is significant, the acceleration of the remaining unstable ice part shows a discontinuity. In that case, the data are not described by Eq. (1) anymore. Other phenomena, like ice melting or strong variations in basal sliding may also affect the acceleration of the unstable ice mass (Pralong and Funk, $2005 \mathrm{~b})$. The time span $\Delta t_{\mathrm{B}}$ used for a prediction should correspond to the last time span prior to the failure during which no external disturbances interfere with the failure process. The reference value $\Delta t_{\mathrm{B}}^{*}$ corresponds to a mean of values observed in practice.

For this analysis, it will be assumed that the relative motion $Y$ of the unstable ice mass is known. Therefore, Eq. (3) (with parameters $\theta_{1}$ to $\theta_{4}$ ) is considered. The parameter transformation as given by Eq. (8) is applied to the four parameters $\theta_{i}$

$$
\begin{aligned}
& \theta_{1}=\max \left(t_{i}\right)+\exp \left(\phi_{1}\right), \quad \theta_{2}=1-\exp \left(\phi_{2}\right), \\
& \theta_{3}=\phi_{3}, \quad \theta_{4}=\phi_{4} .
\end{aligned}
$$



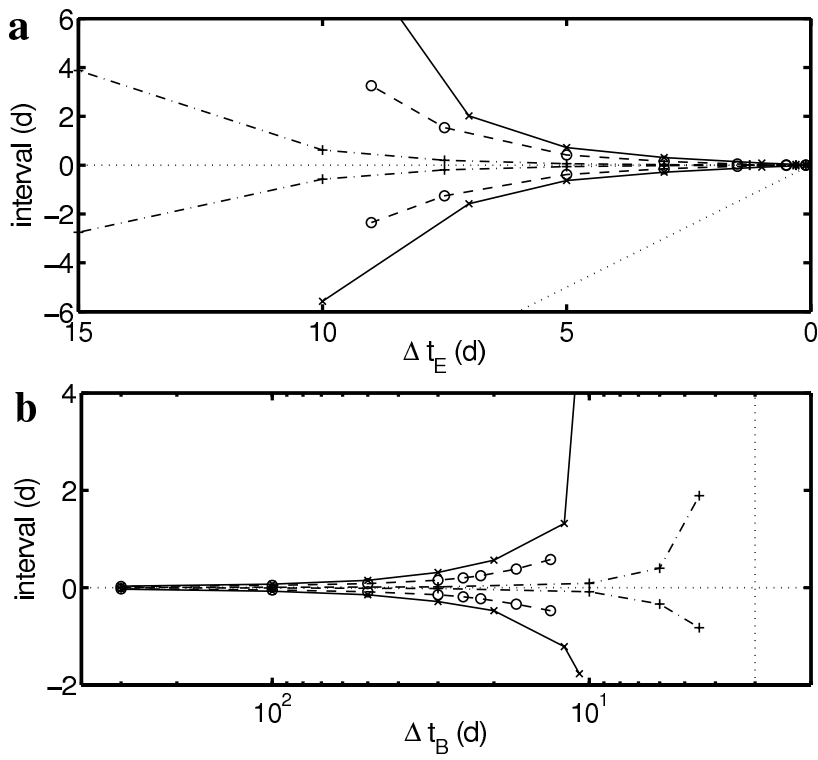

Fig. 5. Influence of the value of $\Delta t_{\mathrm{E}}$ (a) and $\Delta t_{\mathrm{B}}$ (b) on the $95 \%$ confidence interval of $\theta_{1}$. The solid lines with the marks " $\times$ " (the dashed lines with "o" and the dash-dotted lines with "+", respectively) are calculated by analyzing data sets synthesized with Eq. 3 and the values $\hat{\theta}_{i}$ (see Table 1) estimated from the measurements at Gruben (Eiger and $\mathrm{Mönch}_{1}$, respectively). The horizontal dotted lines on both panels correspond to the estimate of $\theta_{1}$. The inclined dotted line in panel a) and the vertical dotted line in panel b) depict the time $t_{\mathrm{E}}$ of the end of the measurements.

\subsection{Results and discussion}

The variations in the $95 \%$ confidence interval for $\theta_{1}$ as a function of $\mathcal{S}_{i}$ are presented in Fig. 5. For the three analyzed break-off events (Gruben, Eiger, Mönch ${ }_{1}$ ), the results are qualitatively similar. The influence of $\sigma_{Y}$ and $\delta_{Y}$ is here similar to the case of a linear regression and is not presented (the size of the confidence interval is proportional to $\sigma_{Y}$ and to $\left.\delta_{Y}{ }^{0.5}\right)$. Only the influence of $\Delta t_{\mathrm{E}}$ and $\Delta t_{\mathrm{B}}$ is considered. The size of the confidence interval decreases with decreasing $\Delta t_{\mathrm{E}}$ (Fig. 5a), since the information about the failure time contained in the data sets becomes more accurate as $t_{\mathrm{E}}$ tends to $\theta_{1}$. The size of the confidence interval decreases with increasing $\Delta t_{\mathrm{B}}$ (Fig. 5b), since a longer time span of measurements reduces the effects of the inaccuracy of individual measurements.

The analysis of the profile- $t$ plots shows that the $t$-profile $\tau\left(\phi_{1}\right)$ remains approximately linear for all $\Delta t_{\mathrm{E}}$ and $\Delta t_{\mathrm{B}}$ (for the influence of $\Delta t_{\mathrm{E}}$ on $\tau\left(\phi_{1}\right)$ see Fig. 6). The confidence interval of $\phi_{1}$ is therefore symmetric. The asymmetry of the confidence interval for $\theta_{1}$ observed in Fig. 5 is due to the nonlinearity of the parameter transformation $11_{1}$. The analysis of the profile traces shows that $\Delta t_{\mathrm{E}}$ and $\Delta t_{\mathrm{B}}$ strongly influence the correlation between $\theta_{1}$ and $\theta_{i \neq 1}$ (for $\Delta t_{\mathrm{E}}$, see Fig. 6). The correlation between the parameters strongly influences the size of the confidence interval.
Figure 5a shows that accurate long-term predictions are difficult. The confidence interval of $\theta_{1}$ is small only if the time span between $t_{\mathrm{E}}$ and the time of failure does not exceed a few days. For earlier end points $t_{\mathrm{E}}$, the confidence interval is large and its lower boundary tends toward the line corresponding to the time $t_{\mathrm{E}}$ of the last measurement (the inclined dotted line in Fig. 5a). This suggests that a forecast performed at an early stage of the destabilization can falsely lead to the prediction of an impending failure. This problem was observed by Bufe and Varnes (1993) for the prediction of earthquakes and experienced by the authors during the prediction of the failure of hanging glaciers. To estimate the quality of the prediction of an impending failure, the uncertainty on the forecast has to be determined or/and an a priori value of the parameters $\theta_{2}$ and $\theta_{3}$ has to be approximately known.

For practical application, $\sigma_{Y}$ is constrained by the measurement method, $\Delta t_{\mathrm{E}}$ is continuously decreasing during an ongoing measurement process and $\delta_{Y}$, and $\Delta t_{\mathrm{B}}$ are free parameters. To improve the accuracy of the prediction, $\sigma_{Y}$ can be decreased by considering the mean of repeated measurements or $\delta_{Y}$ simply decreased. However, the measurements at Eiger and Mönch (Figs. 2c and 3c), revealed that the disturbance $Z$ is a correlated noise for time lags of less than one day ${ }^{7}$. For such a noise, measurements with a lag less than one day do not improve the accuracy of the prediction. During a destabilization process, it is attempted to conduct measurements until failure occurs, in order to continuously improve the prediction. However, for technical reasons, the measurements could be interrupted prior to the failure. If $\Delta t_{\mathrm{E}}$ is too large, the prediction is inaccurate. $\Delta t_{\mathrm{B}}$ is chosen as large as possible, but is limited by the presence of external disturbances which may impose the value of $t_{\mathrm{B}}$ (see above).

The differences in the size of the confidence intervals observed in Fig. 5 for the destabilization processes at Gruben, Eiger and Mönch are due to different values of the parameters $\theta_{2}$ and $\theta_{3}$ (Table 1). Figure 7 a shows the influence of the variation in $\theta_{2}$ on the confidence interval of $\theta_{1}$, the parameter $\theta_{3}$ and the parameter set $\mathcal{S}$ remaining constant. The minimum of the confidence interval corresponds to approximately $\theta_{2}=0.6$. Figure $7 \mathrm{~b}$ shows the influence of the variation in $\theta_{3}$ on the confidence interval of $\theta_{1}$, the parameter $\theta_{2}$ and the parameter set $\mathcal{S}$ remaining constant. A large value of $\theta_{3}$ is associated with an important displacement of the unstable ice mass during the failure process; that is, the noise $Z$ becomes small relative to the variations in $Y$ (the size of the confidence interval is proportional to $\theta_{3}^{-1}$ ). Therefore,

\footnotetext{
${ }^{7}$ For the Eiger data set, this is probably due to the daily variations in the basal sliding (see above), which disappear by measuring the relative motion of the unstable ice mass. For the Mönch data set, this is likely due to daily variations in the position of the theodolite. The daily variations apparently have not been entirely removed by the correction of the theodolite position (see Appendix A). For the Gruben data set, where daily variations should theoretically not appear (here, the relative motion of the unstable ice mass is measured), the sparse data does not allow to determine whether $Z$ is a correlated or an uncorrelated noise.
} 
a
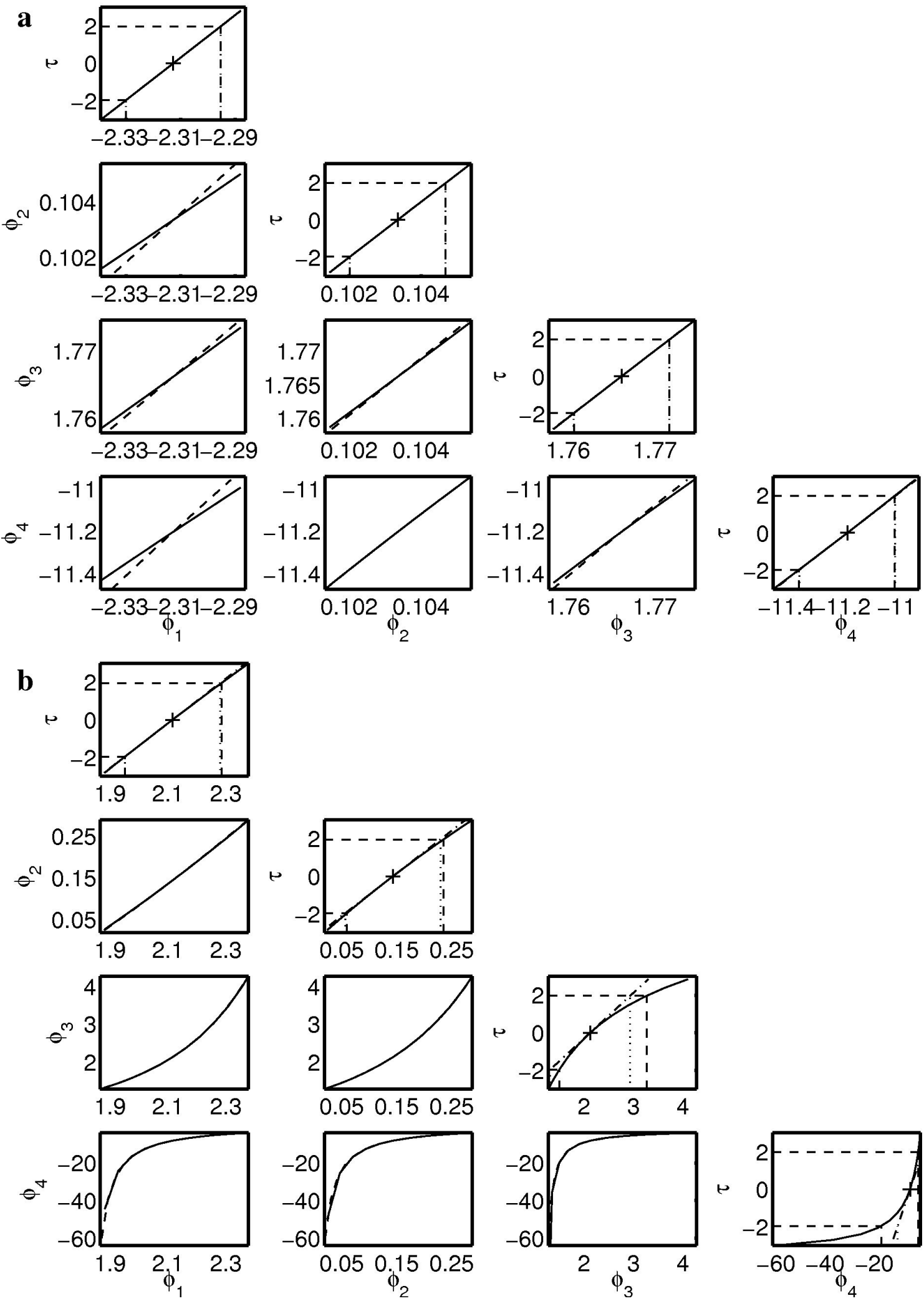

Fig. 6. Influence of $\Delta t_{\mathrm{E}}$ on the profile $t$-plots and profile traces. The non-linear regression analysis is based on two data sets synthesized with Eq. (3) and the value of the Eiger parameters $\theta_{i}$ listed in Table 1 . The value of the parameters $\mathcal{S}_{i}$ is for a) $\mathcal{S}=\left(\sigma_{Y}^{*}, f_{Y}^{*}, \Delta t_{\mathrm{E}}=0.1 \mathrm{~d}, \Delta t_{\mathrm{B}}^{*}\right)$ and for b) $\mathcal{S}=\left(\sigma_{Y}^{*}, f_{Y}^{*}, \Delta t_{\mathrm{E}}=7.5 \mathrm{~d}, \Delta t_{\mathrm{B}}^{*}\right)$, where the reference values (marked with ${ }^{*}$ ) are given by Eq. (10). The pictures show the profile- $t$ plots with $95 \%$ confidence intervals. The parameter transformation is given by Eq. (11). 
larger values of $\theta_{3}$ decrease the confidence interval of $\theta_{1}$. This effect explains the main differences in the size of the confidence interval observed between the different break-off events in Fig. 5.

\section{Log-periodic oscillations}

\subsection{Background}

Log-periodic oscillations are characteristic oscillations which may occur during a critical process leading to a finitetime singularity. They are superimposed on the motion $f(t)$ with a frequency proportional to $\log \left(\theta_{1}-t\right)$. They appear as solutions of Voight's equation when the exponent $\theta_{2}$ becomes complex (Sornette and Sammis, 1995). For $\theta_{2} \neq 0$, the real part $f_{\text {lpo }}(t, \boldsymbol{\theta})$ of the model function with oscillations takes the form (Sornette and Sammis, 1995)

$$
\begin{aligned}
f_{\text {lpo }}(t, \boldsymbol{\theta})= & \theta_{4}-\frac{\theta_{3}}{\theta_{2}}\left(\theta_{1}-t\right)^{\theta_{2}} . \\
& {\left[1+\theta_{6} \sin \left(2 \pi \frac{\log \left(\theta_{1}-t\right)}{\log \left(\theta_{7}\right)}+\theta_{8}\right)\right], }
\end{aligned}
$$

where $\theta_{6}$ is the relative amplitude ${ }^{8}, \theta_{7}$ is the logarithmic periodicity and $\theta_{8}$ is the phase shift of the log-periodic oscillation. An acceleration with oscillations, which is superimposed on a steady motion takes the form

$$
\begin{aligned}
f_{\text {lpo }}^{\mathrm{a}}(t, \boldsymbol{\theta})= & \theta_{5} t+\theta_{4}-\frac{\theta_{3}}{\theta_{2}}\left(\theta_{1}-t\right)^{\theta_{2}} . \\
& {\left[1+\theta_{6} \sin \left(2 \pi \frac{\log \left(\theta_{1}-t\right)}{\log \left(\theta_{7}\right)}+\theta_{8}\right)\right] . }
\end{aligned}
$$

Log-periodic oscillations have been observed for numerous finite-time singularities: fracture of structures, earthquakes, rock bursts, financial crashes (see the review of Zhou and Sornette, 2002) and were suggested by Lüthi (2003) in the case of failure of hanging glaciers. Several attempts have been made to explain log-periodic oscillations. Ide and Sornette (2002) related log-periodic behavior with systems that contain a relaxation mechanism reducing the damage in the system. However, they did not obtain a response from the system corresponding to Eq. (12). Sahimi and Arbabi (1996) related log-periodic behavior to dynamic crack interactions at different scales. The physical meaning of $\theta_{7}$ is not fully revealed by this approach (Lüthi, 2003), either.

\subsection{Observations}

The measurements carried out at the Mönch hanging glacier show log-periodic oscillations: Figure $3 \mathrm{c}$ presents the oscillations isolated from the global acceleration (residuals $R_{i}=Y_{i}^{\mathrm{a}}-f^{\mathrm{a}}\left(t_{i}, \boldsymbol{\theta}\right)$ for a fitted function $f^{\mathrm{a}}$ of the form of Eq. 7) for the material point 1 (Fig. 3a). As expected, the other two points considered (points 2 and 3; Fig. 3a) also present log-periodic oscillations (Figs. 8a and b). For all

\footnotetext{
${ }^{8}$ Although $\theta_{6}$ is a constant value, the amplitude of the logperiodic oscillations varies with time, since the term multiplying the square bracket varies with time. If $\theta_{2}<0$, the amplitude of the oscillations increases until failure; if $\theta_{2}>0$, it decreases.
}
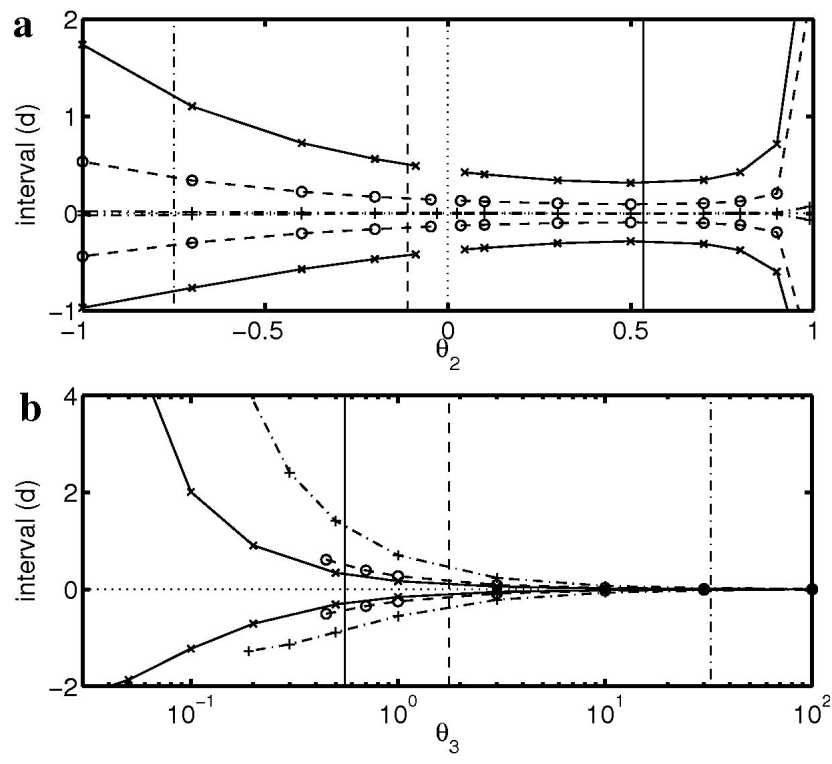

Fig. 7. Influence of the value of $\theta_{2}$ (a) and $\theta_{3}$ (b) on the $95 \%$ confidence interval of $\theta_{1}$. The solid lines marked with " $\times$ " (the dashed line with "o" and the dash-dotted line with "+", respectively) are calculated by analyzing data sets synthesized with the value $\hat{\theta}_{3}$ (for panel a), the value $\hat{\theta}_{2}$ (for panel b) estimated from the measurements at Gruben (Eiger and Mönch $_{1}$, respectively), the values of the parameter set $\mathcal{S}^{*}$ (Eq. 10) and the Eq. (3). The vertical solid lines (dashed and dash-dotted lines, respectively) correspond to the value $\hat{\theta}_{2}$ (for panel a), to the value $\hat{\theta}_{3}$ (for panel $b$ ) estimated from the measurements at Gruben (Eiger and Mönch ${ }_{1}$, respectively). The horizontal dotted lines on both panels correspond to the estimate of $\theta_{1}$. The vertical dotted line in panel a) marks the singularity of Eq. (3) at $\theta_{2}=0$.

three points, the fit of the log-periodic oscillations is mostly in accordance with the smooth curve through the residuals $R_{i}$. In the case of Gruben, the existence of log-periodic oscillations is probable but cannot be verified due to the sparse data (Fig. 1c). For the measurements at Eiger, no oscillations could be observed (Fig. 2c). This, however, does not mean that they do not exist; they might be hidden by the daily variations in the glacier velocity.

The amplitude of the log-periodic oscillations equals

$-\frac{\theta_{3}}{\theta_{2}}\left(\theta_{1}-t\right)^{\theta_{2}} \theta_{6}$

The comparison of the oscillations observed for the three points at Mönch (Fig. 8c) reveals the same amplitude of oscillation even though $\theta_{2}$ and $\theta_{3}$ do not have the same values for the different points 9 . Thus, the parameter $\theta_{6}$ compensates for the different values of $\theta_{2}$ and $\theta_{3}$. $\theta_{6}$ is therefore not constant (Table 1). Figure $8 \mathrm{c}$ also shows that the oscillations are in phase, at least during the period of the measurements. The

\footnotetext{
${ }^{9}$ The global displacement, given by $-\left(\theta_{3} / \theta_{2}\right)\left(\theta_{1}-t\right)^{\theta_{2}}$, has not the same magnitude for points 1,2 and 3 . The global displacement from the beginning to the end of the measurements amounts to $3.2[\mathrm{~m}], 2.95[\mathrm{~m}]$ and $2.4[\mathrm{~m}]$ for point 1,2 and 3, respectively.
} 

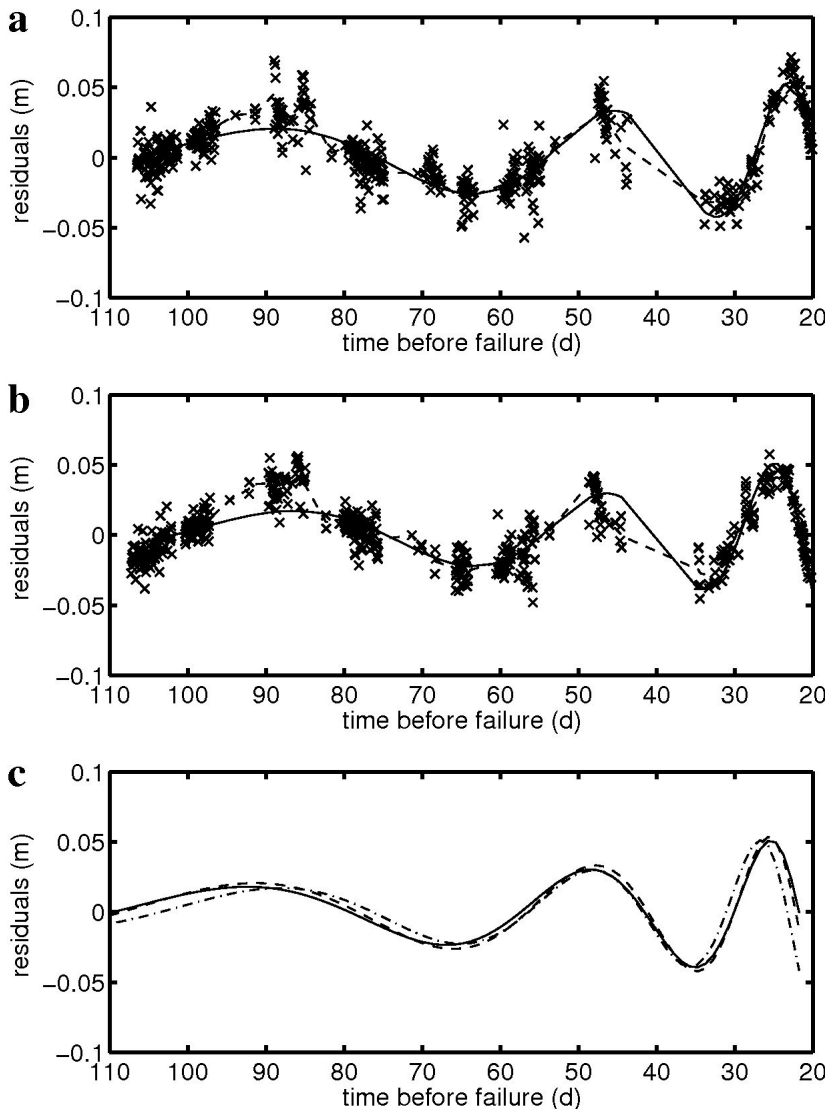

Fig. 8. Log-periodic oscillations observed at Mönch. They correspond to the residuals $Y^{\mathrm{a}}-f^{\mathrm{a}}(t, \boldsymbol{\theta})$ associated with (a) point 2 in Fig. 3a and (b) point 3 in Fig. 3a. The solid lines depict the fit of the log-periodic oscillations. The dashed lines show the smooth curve of the residuals $Y^{\mathrm{a}}-f^{\mathrm{a}}(t, \boldsymbol{\theta})$. (c) Superposition of the three observed oscillations. The solid line refers to $\mathrm{Mönch}_{1}$; the dashed line to Mönch 2 ; the dash-dotted line to Mönch $_{3}$. The values of the oscillations parameters are given in Table 1 .

estimates of the logarithmic wavelength $\theta_{7}$ is similar for the three points, in contrast to the phase shift $\theta_{8}$ (Table 1). The variations of the phase shift compensate for the difference in the estimated values of the failure time $\hat{\theta}_{1}$ reported in Table 1 (lower part of the table).

$\mathrm{A}(H, q)$-analysis can be alternatively used to identify the parameters $\theta_{6}, \theta_{7}, \theta_{8}$ of the log-periodic oscillations (Zhou and Sornette, 2002). In this method, the parameters $\theta_{3}$ and $\theta_{4}$ of the general trend disappear and thus do not need to be identified. The disadvantage of this method is that it requires the estimation of a derivative (the $(H, q)$-derivative of $Y$ ), which magnifies the noise of the data.

\subsection{Predictions}

A prediction from a model with no log-periodic oscillations provides inaccurate results. Figure 9 shows, for the Mönch data set, that $\hat{\theta}_{1}$ strongly varies with $\Delta t_{\mathrm{E}}$ if the fitting function does not include log-periodic oscillations (this effect is reduced for small $\Delta t_{\mathrm{E}}$ ). In contrast, $\hat{\theta}_{1}$ is much less sensitive

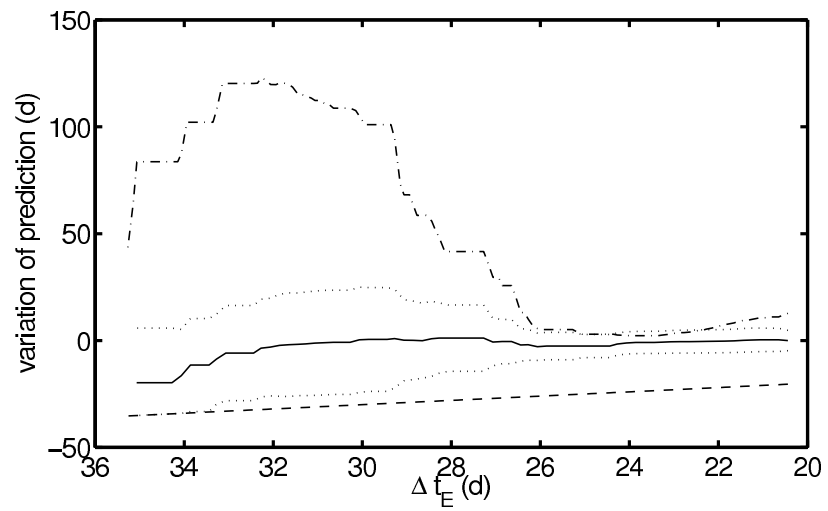

Fig. 9. Influence of the variation of $\Delta t_{\mathrm{E}}$ on the predicted time of failure $\hat{\theta}_{1}$ estimated by fitting the Mönch $_{1}$ data set using the model without log-periodic oscillations (Eq. 7) (dash-dotted line) and with log-periodic oscillations (Eq. 13) (solid line). The $y$-label "variation of prediction" means the difference between $\hat{\theta}_{1}$ estimated by varying $\Delta t_{\mathrm{E}}$ and $\hat{\theta}_{1}$ estimated with Eq. (13) and the complete data set (minimum $\Delta t_{\mathrm{E}}$ ). The two dotted lines show the $95 \%$ confidence interval of the prediction using the model with oscillations. The interval is estimated using an autoregressive moving-average (ARMA) model (e.g. Brockwell and Davis, 2002) which assumes a lagged dependence of the residual terms. The dashed line depicts the time $t_{\mathrm{E}}$ of the end of the measurements. The fits are performed with an imposed value of $\theta_{5}$.

to $\Delta t_{\mathrm{E}}$ when the log-periodic oscillations are taken into account (see also Table 1). The dependence of $\hat{\theta}_{1}$ on $\Delta t_{\mathrm{E}}$ in the former case results from the fact that the last measurements of the time series have the largest influence on the prediction (due to the non-linearity of the function) and that the value of these last measurements varies around the global trend (due to the presence of log-periodic oscillations) when $\Delta t_{\mathrm{E}}$ varies. A positive deviation of the last measurements (Fig. 8) from the global trend leads to an underestimation of the time span until failure, whereas a negative deviation leads to an overestimation.

Figure 9 also illustrates that a forecast performed at an early stage of the destabilization cannot exclude an impending failure, since, with increasing $\Delta t_{\mathrm{E}}$, the confidence interval tends toward the line corresponding to the time of the prediction (dashed line). If the log-periodic oscillations are not considered in the function used for the prediction, the forecast of an impending failure could be erroneous, since the variations observed in the prediction (dash-dotted line) can induce an underestimation of the time span until failure.

\section{Conclusions}

Accurate predictions of the failure of hanging glaciers are only possible when the time between the end of the measurements and the effective time of failure becomes small (order of magnitude of one week). An early monitoring of the failure process increases the accuracy of the prediction, as long as no external disturbances interfere with the failure process 
during the measurement period. The value of the parameters $\theta_{2}$ and $\theta_{3}$ describing the acceleration process strongly influences the accuracy of the forecast, since the model to be fitted is non-linear. If the values of these parameters cannot be estimated in advance, an a priori approximation of the accuracy of the prediction is not possible; it only emerges during the prediction process.

Log-periodic oscillations were clearly observed in one of the three break-off events analyzed. For this event, the amplitude, frequency and phase of the oscillations appear to be spatially homogeneous over the entire unstable ice mass, whereas the shape of the global acceleration is spatially inhomogeneous (this behavior has also been observed recently on a hanging glacier in the north face of Weisshorn). This suggests that the oscillations may result from a global process acting on the entire unstable ice mass. It has been shown subsequently that the influence of the oscillations on the forecast is significant. This implies, if oscillations are observed in a data set, that they must be considered in order to achieve an accurate prediction.

It was not possible to observe disturbances that are intrinsically related to the destabilization process, like the jerky motion observed by Haeberli (1975), since important external disturbances hid this behavior, and the accuracy and frequency of the measurements were not adequate. Further investigations should be carried out to determine the properties of these intrinsic disturbances. Information for maximizing the accuracy of the prediction and minimizing the number of necessary measurements could be gained through this analysis.

The prediction of the effective time of failure is based on the assumption that the failure time parameter $\hat{\theta}_{1}$ corresponds to the effective time of the break-off; that is, the failure occurs at infinite velocity. This assumption is not precise for ductile materials (e.g. ice), since the fracture is usually observed to occur at an earlier stage of the acceleration process, i.e. at a finite velocity (e.g. Lemaitre, 1996). This behavior can be related to the inhomogeneity of the crack net (Pralong and Funk, 2005a, Appendix A). Recent observations performed on a hanging glacier at the Weisshorn north face also suggest that the failure occurs at a finite velocity. Further investigations need to be carried out in order to improve the physical understanding of the failure processes so that an appropriate model of fracture can be obtained.

\section{Appendix A Reconstruction of the theodolite position from measurements}

The measurements carried out at the Mönch hanging glaciers were affected by the variation in the position of the theodolite. The theodolite was installed on the terrace of a building (Sphinx building at Jungfraujoch) which is subject to small oscillations due to thermal constraints in the structure of the building and probably to the motion of the foundations. Four reference points located on rock spurs around the theodolite were installed to reconstruct the position of the theodolite. This reconstruction is an overdetermined inverse problem.

The forward problem, which couples the position $\left(P t_{x}, P t_{y}, P t_{z}\right)$ and the horizontal rotation $\phi$ of the theodolite to the distance $d_{i}$, the $\operatorname{cosine} \cos v_{i}$ of the vertical angle, the cosine $\cos h_{i}$ of the horizontal angle and the $\operatorname{sine} \sin h_{i}$ of the horizontal angle obtained by the measurement of a reference point $i$ ( $i=1, \ldots, m$, with $m$ the number of reference points, here $m=4$ ) in a spherical coordinates system, is given by

$d_{i}=\left(\left(P i_{x}-P t_{x}\right)^{2}+\left(P i_{y}-P t_{y}\right)^{2}+\left(P i_{z}-P t_{z}\right)^{2}\right)^{1 / 2}$, $\cos v_{i}=\frac{P i_{z}-P t_{z}}{d_{i}}$,

$\cos h_{i}=c_{1}\left(\left(P i_{y}-P t_{y}\right)+\left(P i_{x}-P t_{x}\right) \frac{\sin \phi}{\cos \phi}\right)$,

$\sin h_{i}=c_{1}\left(\left(P i_{x}-P t_{x}\right)-\left(P i_{y}-P t_{y}\right) \frac{\sin \phi}{\cos \phi}\right)$,

where $\left(P i_{x}, P i_{y}, P i_{z}\right)$ is the position of the reference point $i$ and

$c_{1}=\left(1+\frac{\sin ^{2} \phi}{\cos ^{2} \phi}\right)^{-1}\left(d_{i} \sin v_{i} \cos \phi\right)^{-1}$,

$\sin v_{i}=\left(1-\cos ^{2} v_{i}\right)^{1 / 2}$.

The referential $(x, y, z)$ has to be chosen such that $\cos \phi \neq 0$. The linearized forward system then reads

$$
\left(\begin{array}{l}
d_{i}-d_{i}^{(0)} \\
\cos v_{i}-\cos ^{(0)} v_{i} \\
\cos h_{i}-\cos ^{(0)} h_{i} \\
\sin h_{i}-\sin ^{(0)} h_{i}
\end{array}\right)=G^{(0)}\left(\begin{array}{l}
P t_{x}-P t_{x}^{(0)} \\
P t_{y}-P t_{y}^{(0)} \\
P t_{z}-P t_{z}^{(0)} \\
\phi-\phi^{(0)}
\end{array}\right),
$$

where the superscript ${ }^{(0)}$ denotes the linearization point, and $G^{(0)}$ is the Jacobian matrix (a $4 m \times 4$ matrix) defined as

$G^{(0)}=\left.G\right|_{\left(P t_{x}^{(0)}, P t_{y}^{(0)}, P t_{z}^{(0)}, \phi^{(0)}\right)}$,

with

$G=\left[\begin{array}{cccc}\frac{\partial d_{i}}{\partial P t_{x}} & \frac{\partial d_{i}}{\partial P t_{y}} & \frac{\partial d_{i}}{\partial P t_{z}} & 0 \\ \frac{\partial \cos v_{i}}{\partial P t_{x}} & \frac{\partial \cos v_{i}}{\partial P t_{y}} & \frac{\partial \cos v_{i}}{\partial P t_{z}} & 0 \\ \frac{\partial \cos h_{i}}{\partial P t_{x}} & \frac{\partial \cos h_{i}}{\partial P t_{y}} & \frac{\partial \cos h_{i}}{\partial P t_{z}} & \frac{\partial \cos h_{i}}{\partial \phi} \\ \frac{\partial \sin h_{i}}{\partial P t_{x}} & \frac{\partial \sin h_{i}}{\partial P t_{y}} & \frac{\partial \sin h_{i}}{\partial P t_{z}} & \frac{\partial \sin h_{i}}{\partial \phi}\end{array}\right]$.

The system (A3) is inverted in the sense of the least-squares approach. The inverse system reads

$\left(\begin{array}{l}P t_{x}^{(n+1)} \\ P t_{y}^{(n+1)} \\ P t_{z}^{(n+1)} \\ \phi^{(n+1)}\end{array}\right)=\left(\begin{array}{l}P t_{x}^{(n)} \\ P t_{y}^{(n)} \\ P t_{z}^{(n)} \\ \phi^{(n)}\end{array}\right)$

$+A^{(n)}\left(\begin{array}{lc}d_{i}^{(n+1)} & -d_{i}^{(n)} \\ \cos ^{(n+1)} v_{i}-\cos ^{(n)} v_{i} \\ \cos ^{(n+1)} h_{i}-\cos ^{(n)} h_{i} \\ \sin ^{(n+1)} h_{i}-\sin ^{(n)} h_{i}\end{array}\right)$.

The superscripts $(n+1),{ }^{(n)}$ denote the solution at the $(n+1)$ th iteration and $n$th iteration, respectively. $d_{i}^{(n)}, \cos ^{(n)} v_{i}$, 
$\cos ^{(n)} h_{i}$ and $\sin ^{(n)} h_{i}$ are evaluated with Eq. (A1) at $P t_{x}^{(n)}$, $P t_{y}^{(n)}, P t_{z}^{(n)}$ and $\phi^{(n)}$, and the $4 \times 4 m$ matrix $A^{(n)}$ is given by

$A^{(n)}=\left(G^{(n)}{ }^{T} G^{(n)}\right)^{-1} G^{(n)^{T}}$,

where the Jacobian matrix $G^{(n)}$ reads

$G^{n}=\left.G\right|_{\left(P t_{x}^{(n)}, P t_{y}^{(n)}, P t_{z}^{(n)}, \phi^{(n)}\right)}$.

The system (A6) is iteratively solved until the relative difference between the solution of the $(n+1)$ th and $n$th iterations becomes less than $10^{-6}$

The standard deviation of the displacement of the theodolite in east, north and vertical directions amounts to 0.7 , 1.1 and $4 \mathrm{~mm}$ and the standard deviation of the rotation to $9 \times 10^{-3}$ degrees.

\section{Appendix B Profile- $t$ plots and profile traces}

The sum of squares of residuals is

$S(\boldsymbol{\theta})=\sum_{i=1}^{n}\left(y_{i}-f\left(t_{i}, \boldsymbol{\theta}\right)\right)^{2}$,

where $f$ is the model function, $\boldsymbol{\theta}$ are the parameters of $f, n$ is the number of measurements and $y_{i}$ is the measurements at time $t_{i}$. Let $\hat{\boldsymbol{\theta}}_{\mid k}\left(\theta_{k}\right)$ be the vector of parameters that minimizes $S(\boldsymbol{\theta})$ subject to a given value $\theta_{k}$. The likelihood ratio test statistic for a null hypothesis about $\theta_{k}$ alone is

$\tilde{S}_{k}\left(\theta_{k}\right)=(n-p) \frac{S\left(\hat{\boldsymbol{\theta}}_{\cdot \mid k}\left(\theta_{k}\right)\right)-S(\hat{\boldsymbol{\theta}})}{S(\hat{\boldsymbol{\theta}})}$,

where $p$ is the number of parameters of $\boldsymbol{\theta}$. For linear regressions, $\tilde{S}_{k}\left(\theta_{k}\right)$ is a quadratic function. The signed square root of $\tilde{S}_{k}$

$\tau_{k}\left(\theta_{k}\right)=\operatorname{sign}\left(\theta_{k}-\hat{\theta}_{k}\right)\left(\tilde{S}_{k}\left(\theta_{k}\right)\right)^{\frac{1}{2}}$

is called the $t$-profile. It is linear for a linear regression function. The nonlinearity of $\tau_{k}$ therefore reflects the nonlinearity of the regression function around the best-fitting parameter.

Confidence intervals for a single parameter $\theta_{k}$ can be read off the $t$-profile plot by intersecting horizontal lines at $\pm q_{1-\alpha}^{t_{n-p}}$ with the $t$-profile and determining the respective $\theta_{k}$ values. $q_{1-\alpha}^{t_{n-p}}$ is the $1-\alpha$ quantile of the $t$-distribution with $n-p$ degrees of freedom.

The functions $\hat{\theta}_{j \mid k}\left(\theta_{k}\right)$ are called the profile traces of $\theta_{k}$. For plotting, the profile traces $\hat{\theta}_{j \mid k}\left(\theta_{k}\right)$ and $\hat{\theta}_{k \mid j}\left(\theta_{j}\right)$ are superimposed in the same panel. The axes for the two curves must have identical meaning, which means that for one of the curves, the argument is plotted in a vertical direction and the result in a horizontal one.

For more informations about profile- $t$ plots and profile traces, the reader is invited to consult Bates and Watts (1988).
Acknowledgements. The authors want to thank A. Helmstetter for her comments and G. Zoeller for his editing work. They are also grateful to the Jungfrau Railways in Interlaken and the Jungfraujoch research station for their support for the field work on Eiger and Mönch.

Edited by: G. Zoeller

Reviewed by: A. Helmstetter and another referee

\section{References}

Amitrano, D., Grasso, J.-R., and Senfaute, G.: Seismic precursory patterns before a cliff collapse and critical point phenomena, Geophys. Res. Lett., 32, L08 314, doi:10.1029/2004GL022 270, 2005.

Bates, D. M. and Watts, D. G.: Nonlinear Regression Analysis and Its Applications, John Wiley \& Sons, New York, 1988.

Bowman, D. D., Ouillon, G., Sammis, C. G., Sornette, A., and Sornette, D.: An observational test of the critical earthquake concept, J. Geophys. Res., 103, 24 359-24 372, 1998.

Brockwell, P. J. and Davis, R. A.: Introduction to time series and forecasting, Springer, New York, 2002.

Bufe, C. G. and Varnes, D. J.: Predictive Modeling of the Seismic Cycle of the Greater San Francisco Bay Region, J. Geophys. Res., 98, 9871-9883, 1993.

Crosta, G. B. and Agliardi, F.: Failure forecast for large rock slides by surface displacement measurements, Can. Geotech. J., 40, 176-191, 2003.

Flotron, A.: Movement studies on hanging glaciers in relation with an ice avalanche, J. Glaciol., 19, 671-672, 1977.

Haeberli, W.: Überwachung von Kalbungsflutwellen am Grubengletscher, Wallis, Schweizerische Bauzeitung, 93, 694-696, 1975.

Helmstetter, A., Sornette, D., and Grasso, J.-R.: Mainshocks are aftershocks of conditional foreshocks: How do foreshock statistical properties emerge from aftershock laws, J. Geophys. Res. 108, 2046, doi:10.1029/2002JB001 991, 2003.

Hock, R. and Noetzli, C.: Areal melt and discharge modelling of Storglaciären, Sweden, Ann. Glaciol., 24, 211-217, 1997.

Ide, K. and Sornette, D.: Oscillatory finite-time singularities in finance, population and rupture, Physica A-Statistical Mechanics and its Applications, 307, 63-106, 2002.

Iken, A.: Movement of a large ice mass before breaking off, J. Glaciol., 19, 565-605, 1977.

Johansen, A. and Sornette, D.: Critical ruptures, European Physical Journal B, 18, 163-181, 2000.

Johansen, A. and Sornette, D.: Finite-time singularity in the dynamics of the world population, economic and financial indices, Physica A, 294, 465-502, 2001.

Kachanov, L. M.: Time of the rupture process under creep conditions (in Russian), Izv. Akad. Nauk. USSR, Otd. Tekh. Nauk. 26-31, 1957.

Lemaitre, J.: A course on damage mechanics, Springer, Berlin, Germany, 228 p., 1996.

Lüthi, M.: Instability in glacial systems, in Milestones in Physical Glaciology: From the Pioneers to a Modern Science, Mitteilungen, VAW, ETH-Zürich, 180, 63-70, 2003.

Lyakhovsky, V., Ben-Zion, Y., and Agnon, A.: Earthquake cycle, fault zones, and seismicity patterns in a rheologically layered lithosphere, J. Geophys. Res., 106, 4103-4120, 2001. 
Mahrenholtz, O. and Wu, Z.: Determination of Creep Damage Parameters for Polycrystalline Ice, Advances in Ice Technology (3rd Int. Conf. Ice Tech./Cambridge USA), 181-192, 1992.

Pralong, A. and Funk, M.: Dynamic Damage Model of Crevasse Opening and Application to Glacier Calving, J. Geophys. Res., 110, doi:10.1029/2004JB003104, 2005a.

Pralong, A. and Funk, M.: On the stability of hanging glaciers, J. Glaciol., in press, 2005b.

Rabotnov, Y. N.: Creep problems in structural members, NorthHolland, 822 p., 1969.

Röthlisberger, H.: Eislawinen und Ausbrüche von Gletscherseen, in P. Kasser (Ed.), Gletscher und Klima - glaciers et climat, Jahrbuch der Schweizerischen Naturforschenden Gesellschaft, wissenschaftlicher Teil 1978, Birkhäuser Verlag Basel, Boston, Stuttgart, 170-212, 1981.

Sahimi, M. and Arbabi, S.: Scaling Laws for Fracture of Heterogeneous Materials and Rock, Phys. Rev. Lett., 77, 3689-3692, 1996.

Sammis, C. G. and Sornette, D.: Positive feedback, memory, and the predictability of earthquakes, in Self-Organized Complexity in the Physical, Biological, and Social Sciences, National Academy of Sciences, 99, (suppl. 1), 2501-2508, 2002.

Sornette, D. and Sammis, C. G.: Complex critical exponents from renormalization-group theory of earthquakes - implications for earthquake predictions, Journal de Physique I, 5, 607-619, 1995.
Sornette, D., Takayasu, H., and Zhou, W. X.: Finite-time singularity signature of hyperinflation, Physica A, 325, 492-506, 2003.

Sugiyama, S. and Gudmundsson, G. H.: Diurnal variations in vertical strain observed in a temperate valley glacier, Geophys. Res. Lett., 30, 1090, doi:10.1029/2002GL016160, 2003, 2003.

Szyszkowski, W. and Glockner, P. G.: On a multiaxial constitutive Law for Ice, Mechanics of Materials, 5, 49-71, 1986.

Varnes, D. J.: Time-deformation relations in creep to failure of earth materials, in 7th Southeast Asian Geotechnical Conference, Southeast Asian Geotechnical Society, 2, 107-130, 1983.

Voight, B.: A method for prediction of volcanic eruptions, Nature, 332, 125-130, 1988.

Voitkovskii, K. F.: The mechanical Properties of Ice, Izdatel'stvo Akademii Nauk SSSR (in Russian), Trans. AMS-T-R391, American Meteorological Society, Office of Technical Services, US Department of Commerce, Washington, 99 pp., 1960.

Zhou, W. X. and Sornette, D.: Generalized $\mathrm{q}$ analysis of $\log$ periodicity: Applications to critical ruptures, Physical Review E, 66, art. no. 046111, Part 2, 2002.

Zöller, G. and Hainzl, S.: A systematic spatiotemporal test of the critical point hypothesis for large earthquakes, Geophys. Res. Lett., 29, 1558, doi:10.1029/2002GL014 856, 2002. 